\title{
UNIFORM BOUNDEDNESS PRINCIPLES FOR REGULAR BOREL VECTOR MEASURES
}

\author{
JOSEPH KUPKA
}

(Received 22 June 1979)

Communicated by W. Moran

\begin{abstract}
The setting is a compact Hausdorff space $\Omega$. The notion of a Wells class of subsets of $\Omega$ is defined via strange axioms-axioms whose justification rests with examples such as the collection of regular open sets or the range of a strong lifting. A variant of Rosenthal's famous lemma which applies directly to Banach space-valued measures is established, and it is used to obtain, in elementary fashion, the following two uniform boundedness principles: (1) The Nikodym Boundedness Theorem. If $\mathscr{K}$ is a family of regular Borel vector measures on $\Omega$ which is pointwise bounded on every set of a fixed Wells class, then $\mathscr{K}$ is uniformly bounded. (2) The Nikodym Convergence Theorem. If $\left\{\mu_{n}\right\}$ is a sequence of regular Borel vector measures on $\Omega$ which is convergent on every set of a fixed Wells class, then the $\mu_{n}$ are uniformly countably additive, the sequence $\left\{\mu_{n}\right\}$ is convergent on every Borel subset of $\Omega$, and the pointwise limit constitutes a regular Borel measure.
\end{abstract}

1980 Mathematics subject classification (Amer. Math. Soc.): 28-01, 28 A 33, 28 B 05, 28 C 15.

\section{Introduction}

The mission of this paper is to liberate the results of Wells (1969) from their association with the notion of weak convergence of sequences of measures, and from their consequent dependence upon the scalar values of these measures. In so doing we achieve general uniform boundedness principles for Banach spacevalued regular Borel measures. The proofs of these results will also be liberated from any dependence upon the theory of Banach spaces as such. (Not even linear functionals will be used.) The proofs will not only, therefore, become totally elementary, but they will also become easily adaptable, so as to yield alternate proofs of the standard uniform boundedness principles (by which we mean the Nikodym Boundedness Theorem (Diestel and Uhl (1977), Theorem 1, p. 14) and what we shall call the Nikodym Convergence Theorem (Diestel and Uhl (1977), 
Theorem 8, p. 23)), as well as alternate proofs of the variants of these results for fields with the interpolation property (Seever (1968), Theorems C and D, p. 268). (We draw the reader's attention to the fact that, from the (elementary) results of Traynor (1973) (compare with Diestel and Uhl (1977), Corollary 6, p. 29), the Vitali-Hahn-Saks Theorem can be regarded, under circumstances far more general than those considered here, as just a variant of the Nikodym Convergence Theorem. It will consequently receive no further mention in this paper.)

We use the phrase 'uniform boundedness principle' in a loose way to refer to any theorem which asserts a uniformity condition from a 'pointwise' condition. In the measure theoretic setting such theorems normally require a family of measures to be well behaved (in some sense) on each individual set in their common domain. Our overall aim in this paper is to investigate the dividends which can be obtained from the additional assumption that these measures constitute regular Borel measures on a compact Hausdorff space. Under this assumption it will suffice to require only that the given family of measures be well behaved on some of the sets in their common domain, specifically on what we shall call a Wells class (after Wells (1969), Theorem 3, p. 125).

For the definition below, and for the remainder of the paper, we fix the following notation: $\Omega$ denotes a compact Hausdorff space; $\varnothing$ denotes the empty set; $\mathscr{B}$ denotes the collection of Borel subsets of $\Omega$; and int $(A), \operatorname{cl}(A)$ and $A^{c}$ denote, respectively, the interior, the closure and the complement of an arbitrary subset $A$ of $\Omega$.

1.1. Definition. A collection $\mathscr{W}$ of Borel subsets of $\Omega$ will be called a Wells class if it satisfies the following five conditions:

(1.1.1) If $E, F \in \mathscr{W}$, then $E \cap F \in \mathscr{W}$.

(1.1.2) If $E, F \in \mathscr{W}$, and if $\operatorname{cl}(E) \cap \operatorname{cl}(F)=\varnothing$, then $E \cup F \in \mathscr{W}$.

(1.1.3) If $E \in \mathscr{W}$, and if $E$ is not open, then $E^{c} \in \mathscr{W}$.

(1.1.4) If $K$ is a compact subset of $\Omega$, and if $U \supset K$ is an open subset of $\Omega$, then there exists a set $E \in \mathscr{W}$ such that $K \subset E \subset U$.

(1.1.5) If $\left\{E_{n}\right\}$ is an increasing and $\left\{F_{n}\right\}$ a decreasing sequence in $\mathscr{W}$ such that $E_{n} \subset F_{n}$ for all $n$, then there exists a set $E \in \mathscr{W}$ such that $E_{n} \subset E \subset F_{n}$ for all $n$. Property (1.1.5) will be called the interpolation property, and the set $E$ described therein will be said to interpolate the sequences $\left\{E_{n}\right\}$ and $\left\{F_{n}\right\}$. /I

An interpolating set $E$ serves as a substitute for the union of the $E_{n}$, and the arguments of Section 4 (where the interpolation property comes into play) can be rendered considerably less delicate for any Wells class which is closed under the formation of countable (increasing) unions. The remaining properties of a Wells class serve mainly to ensure that the interpolation property can be employed effectively. While we do not follow Wells in requiring that the sets in a Wells class 
be open, the presence of nonopen sets virtually forces the Wells class to be a field, so that the list of known (interesting) Wells classes increases only slightly.

1.2. EXAMPLEs. Each collection of subsets of $\Omega$ listed below is readily shown to satisfy the Wells class axioms.

(1.2.1) The collection $\mathscr{B}$ of Borel sets.

(1.2.2) The collection of open sets.

(1.2.3) The collection of Baire sets.

(1.2.4) The collection of open $F_{\sigma}$ sets.

(1.2.5) The collection of regular open sets (Wells (1969), Theorem 4, p. 128).

(1.2.6) The range of any strong lifting (Dinculeanu (1967), Definition 3 and Proposition 4, p. 406; pp. 201-202). //

We shall frequently strengthen assumption (1.1.4) to read as follows:

1.3. LemMA. Let $\mathscr{W}$ be a Wells class, let $K \subset \Omega$ be compact, and let $U \supset K$ be open. Then there exists a set $E \in \mathscr{W}$ such that $K \subset \operatorname{int}(E) \subset \mathrm{cl}(E) \subset U$.

Proof. From the normality of $\Omega$ we obtain open sets $V_{1}$ and $V_{2}$ such that

$$
K \subset V_{1} \subset \operatorname{cl}\left(V_{1}\right) \subset V_{2} \subset \mathrm{cl}\left(V_{2}\right) \subset U .
$$

Any set $E \in \mathscr{W}$ such that $\operatorname{cl}\left(V_{1}\right) \subset E \subset V_{2}$ is clearly as desired. //

The remainder of the paper is organized as follows: Section 2 sets out the basic notation and terminology; Section 3 presents characterizations of regularity and uniform regularity which serve to illuminate these concepts and to assist with the proofs of the uniform boundedness principles; Section 4 is devoted to a variant of Rosenthal's famous lemma (Diestel and Uhl (1977), Lemma 1, p. 18) which is directly applicable to vector measures, and which will play a crucial role in proving both uniform boundedness principles; finally, Section 5 treats the principles themselves, along with two immediate applications.

\section{Notation and terminology}

Some notation has already been set out in the paragraph preceding Definition 1.1. It remains to explain the generic symbol $\mu$, which, with or without subscripts, will be exclusively reserved to denote a measure. All measures will be either scalar, by which we mean complex-valued, or vector, by which we mean assuming values in a fixed but nameless Banach space whose norm is denoted, as usual, by $\|\cdot\|$. And except for explicit mention to the contrary, all measures will be (norm) 
countably additive. By the semivariation of a (vector) measure $\mu$ with domain $\mathscr{A}$ we shall mean the function $\|\mu\|$ on $\mathscr{A}$ defined by the identity

$$
\|\mu\|(A)=\sup \{\|\mu(H)\|: H \in \mathscr{A} ; H \subset A\}
$$

for all $A \in \mathscr{A}$. The set $A$ is $\mu$-null (or just null) if $\|\mu\|(A)=0$. It should be noted that this is not the standard definition of semivariation (Diestel and Uhl (1977), Definition 4, p. 2), but by Proposition 11, p. 4 of Diestel and Uhl (1977), the two definitions are equivalent in the 'norm' sense, and so are interchangeable for all definitions and results of this paper. We prefer the definition above for this work because it will simplify a few technical arguments (for example, the proof of Proposition 3.2), and because it possesses a natural analogue in more abstract settings (specifically, the ' $h((A))$ ' concept of Traynor (1973), p. 356), to which (some of) the results of this paper may eventually be generalized.

A Borel measure is a measure whose domain is the collection $\mathscr{B}$ of Borel sets. A Borel measure $\mu$ is regular on a set $B$ (in $\mathscr{B}$ ), if, for every number $\varepsilon>0$, there exists a compact set $K \subset B$ and an open set $U \supset B$ such that $\|\mu\|(U \backslash K)<\varepsilon$. A family $\mathscr{K}$ of Borel measures is uniformly regular on $B$ if the above statement holds with the phrase 'for all $\mu \in \mathscr{K}$ ' placed at the end. If $\mathscr{S} \subset \mathscr{B}$, then $\mu$ is regular on $\mathscr{S}$ if $\mu$ is regular on every set $B \in \mathscr{S}$, and $\mu$ is (just plain) regular if it is regular on $\mathscr{B}$. Analogous terminology attaches to uniform regularity.

Uniform regularity of the family $\mathscr{K}$ turns out to be equivalent to uniform countable additivity, which means: for every decreasing sequence $\left\{B_{n}\right\}$ in $\mathscr{B}$ with empty intersection, the $\operatorname{limit}_{\lim _{n \rightarrow \infty}} \mu\left(B_{n}\right)=0$ is uniform for $\mu \in \mathscr{K}$.

Finally, let us deciare that a sequence $\left\{A_{n}\right\}$ of subsets of $\Omega$ is topologically disjoint if we have

$$
\operatorname{cl}\left(A_{n}\right) \cap \mathrm{cl}\left(\bigcup_{i \neq n} A_{i}\right)=\varnothing
$$

for all $n$. This concept was treated, but not named, in Wells (1969).

\section{The notion of regularity}

This section contains a few basic facts about regular Borel vector measures which will illuminate the concept of regularity and provide a foundation for the subsequent material.

The standard measure theoretic uniform boundedness principles apply to finitely additive (albeit 'strongly' additive) measures. Let us note first that noncountably additive measures can never arise in the present setting.

3.1. Proposition. A finitely additive regular Borel vector measure is necessarily countably additive. 
Proof. A most elegant proof, but not the statement, of this fact is (essentially) to be found on pp. 158-159 of Diestel and Uhl (1977) (compare with Dunford and Schwartz (1958), Theorem 13, p. 138). //

The determination of regularity on $\mathscr{B}$ by regularity on a generating set for $\mathscr{B}$ seems not to have been noted in the vector case and follows immediately from the next result.

3.2. Proposirion. The collection of regular sets for a (countably additive) Borel vector measure $\mu$ constitutes a $\sigma$-algebra.

PROof. Closure under the formation of complements is trivial. Closure under the formation of countable intersections follows via a straightforward (and standard) $\varepsilon / 2^{n}$ argument, once it is observed that we have

$$
\|\mu\|(B)=\lim _{n \rightarrow \infty}\|\mu\|\left(B_{n}\right)
$$

for any monotone sequence $\left\{B_{n}\right\}$ in $\mathscr{B}$ whose limit is $B$. This fact, in turn, is readily deduced from the obvious analogues for countably additive measures of Criteria (3) and (7), p. 364, in Traynor (1973). //

3.3. Questions. We have been unable to answer either of the two questions below.

(3.3.1) In view of Proposition 3.1, is the assumption of countable additivity necessary in Proposition 3.2?

(3.3.2) Does the collection of uniformly regular sets for a family $\mathscr{K}$ of regular Borel vector measures constitute a $\sigma$-algebra? //

The affirmative answer to (3.3.2) when open sets are known to be uniformly regular makes up part of the next proposition, which is the principal result of this section.

3.4. Proposition. Let $\mathscr{K}$ be a family of regular Borel vector measures. Then the following assertions are equivalent.

(3.4.1) There exists a Wells class $\mathscr{W}$ such that, for every topologically disjoint sequence $\left\{E_{n}\right\}$ in $\mathscr{W}$, we have $\mu\left(E_{n}\right) \rightarrow 0$ uniformly for $\mu \in \mathscr{K}$.

(3.4.1*) Statement (3.4.1) with ' $\|\mu\|\left(E_{n}\right) \rightarrow 0$ ' in place of ' $\mu\left(E_{n}\right) \rightarrow 0$ '.

(3.4.2) There exists a Wells class $\mathscr{W}$ such that, for every sequence $\left\{E_{n}\right\}$ in $\mathscr{W}$ satisfying $\mathrm{cl}\left(E_{n}\right) \subset \operatorname{int}\left(E_{n+1}\right)$ for all $n$, the sequences $\left\{\mu\left(E_{n}\right)\right\}$ converge uniformly for $\mu \in \mathscr{K}$.

(3.4.2*) Statement (3.4.2) with ' $\mathrm{cl}\left(E_{n+1}\right) \subset \operatorname{int}\left(E_{n}\right)$ ' in place of ' $\mathrm{cl}\left(E_{n}\right) \subset \operatorname{int}\left(E_{n+1}\right)$ '. (3.4.3) $\mathscr{K}$ is uniformly regular on the collection of open subsets of $\Omega$. 
(3.4.3*) $\mathscr{K}$ is uniformly regular on the collection of compact subsets of $\Omega$.

(3.4.4) $\mathscr{K}$ is uniformly regular.

(3.4.5) $\mathscr{K}$ is uniformly countably additive.

Proof. This result constitutes a generalization and extension of Lemma 13, p. 157, in Diestel and Uhl (1977), and, provided that two observations are made, all of the arguments of that lemma may be adapted to this setting. First, one must replace the total variation of each measure by the semivariation, the countable subadditivity of which suffices for present purposes. Second, one must perform a small finesse with Wells classes, a finesse which we shall refer to as the normalityregularity stunt, and which we illustrate simply and completely by establishing the implication $(3.4 .1) \Rightarrow\left(3.4 .1^{*}\right)$.

Suppose that the Wells class $\mathscr{W}$ of $(3.4 .1)$ fails to satisfy $\left(3.4 .1^{*}\right)$. Then we can specify a topologically disjoint sequence $\left\{E_{n}\right\}$ in $\mathscr{W}$, a sequence $\left\{\mu_{n}\right\}$ in $\mathscr{K}$, and a number $\varepsilon>0$ such that $\left\|\mu_{n}\right\|\left(E_{n}\right) \geqslant 3 \varepsilon$ for all $n$. From the normality of $\Omega$ and the topological disjointness of the $E_{n}$, we obtain disjoint open sets $U_{1}$ and $V$ such that $E_{1} \subset U_{1}$, and such that $\operatorname{cl}\left(\bigcup_{n=2}^{\infty} E_{n}\right) \subset V$. We repeat this procedure within $V$ for $E_{2}$ in place of $E_{1}$, and we continue ad infinitum until a sequence $\left\{U_{n}\right\}$ of pairwise disjoint open sets is produced such that $E_{n} \subset U_{n}$ for all $n$. Of course we still have $\left\|\mu_{n}\right\|\left(U_{n}\right) \geqslant 3 \varepsilon$ for each fixed $n$; therefore, we may invoke our definition of semivariation along with the regularity of $\mu_{n}$ to produce a compact set $K_{n} \subset U_{n}$ such that $\left\|\mu_{n}\left(K_{n}\right)\right\| \geqslant 2 \varepsilon$. An application of normality and regularity together will now yield an open set $V_{n}$ such that $K_{n} \subset V_{n} \subset \operatorname{cl}\left(V_{n}\right) \subset U_{n}$, and such that $\left\|\mu_{n}\right\|\left(V_{n} \backslash K_{n}\right)<\varepsilon$. Lastly, we obtain from property (1.1.4) a set $F_{n} \in \mathscr{W}$ such that $K_{n} \subset F_{n} \subset V_{n}$. It is readily verified that the sequence $\left\{F_{n}\right\}$ is topologically disjoint, so that we violate (3.4.1) by noting the inequalities $\left\|\mu_{n}\left(F_{n}\right)\right\| \geqslant\left\|\mu_{n}\left(K_{n}\right)\right\|-\left\|\mu_{n}\right\|\left(V_{n} \backslash K_{n}\right) \geqslant \varepsilon$, which are valid for all $n$.

When Lemma 13, p. 157, of Diestel and Uhl (1977) is adapted to this setting, we obtain the equivalence of all the statements in the present proposition except for (3.4.2) and $\left(3.4 .2^{*}\right)$, which are essentially new. So we complete the proof by establishing

$$
(3.4 .1) \Rightarrow(3.4 .2) \Rightarrow(3.4 .3)
$$

The corresponding implications

$$
(3.4 .1) \Rightarrow\left(3.4 .2^{*}\right) \Rightarrow\left(3.4 .3^{*}\right)
$$

may be established by arguments which are similar in spirit and simpler in execution. It is perhaps curious that the equivalence of (3.4.2) and (3.4.2*) should not appear to be as trivial as that of (3.4.3) and (3.4.3*).

Suppose that the Wells class $\mathscr{W}$ of (3.4.1) fails to satisfy (3.4.2). Then we can 
specify a sequence $\left\{E_{n}\right\}$ in $\mathscr{W}$ and a number $\varepsilon>0$ such that $\operatorname{cl}\left(E_{n}\right) \subset \operatorname{int}\left(E_{n+1}\right)$ for all $n$, and such that, for each $n$, there exist indices $i>j \geqslant n$ and a measure $\mu \in \mathscr{K}$ such that $\left\|\mu\left(E_{i} \backslash E_{j}\right)\right\|=\left\|\mu\left(E_{i}\right)-\mu\left(E_{j}\right)\right\| \geqslant \varepsilon$. Now we have only to note that

$$
\left\|\mu\left(E_{i} \backslash E_{j}\right)\right\| \leqslant\|\mu\|\left(\operatorname{int}\left(E_{i+1}\right) \backslash \mathrm{cl}\left(E_{j-1}\right)\right),
$$

so that the obvious induction procedure will yield a sequence $\left\{U_{n}\right\}$ of pairwise disjoint open sets and a sequence $\left\{\mu_{n}\right\}$ in $\mathscr{K}$ such that $\left\|\mu_{n}\right\|\left(U_{n}\right) \geqslant \varepsilon$ for all $n$. The usual contradiction is then obtained via the normality-regularity stunt.

Finally, we derive (3.4.3) from (3.4.2). Suppose that $\mathscr{K}$ is not uniformly regular on a fixed open set $U$. Then we can specify a number $\varepsilon>0$ such that, for every compact set $K \subset U$, there exists a measure $\mu \in \mathscr{K}$ such that $\|\mu\|(U \backslash K) \geqslant 5 \varepsilon$. The heart of the argument consists in finding, for a given set $E \in \mathscr{W}$ satisfying $\operatorname{cl}(E) \subset U$, a set $F \in \mathscr{W}$ such that $\operatorname{cl}(E) \subset \operatorname{int}(F) \subset \operatorname{cl}(F) \subset U$, and a measure $\mu \in \mathscr{K}$ such that $\|\mu(F)-\mu(E)\|=\|\mu(F \backslash E)\| \geqslant \varepsilon$. The obvious induction procedure based upon this construction will yield a sequence in $\mathscr{W}$ which satisfies the hypotheses but not the conclusion of (3.4.2). So to obtain $F$ and $\mu$, we begin by specifying $\mu \in \mathscr{K}$ to satisfy $\|\mu\|(U \backslash \operatorname{cl}(E)) \geqslant 5 \varepsilon$. Now we must distinguish two cases, depending upon where the mass of $\mu$ is concentrated. Suppose first that $\|\mu(\mathrm{cl}(E) \backslash E)\| \geqslant 2 \varepsilon$. In this case we merely invoke Lemma 1.3 and the regularity of $\mu$ to find a set $F \in \mathscr{W}$ such that $\operatorname{cl}(E) \subset \operatorname{int}(F) \subset \operatorname{cl}(F) \subset U$, and such that $\|\mu\|(F \backslash \mathrm{cl}(E))<\varepsilon$. Then we have

$$
\begin{aligned}
&\|\mu(F \backslash E)\|=\|\mu(F \backslash \operatorname{cl}(E))+\mu(\operatorname{cl}(E) \backslash E)\| \geqslant\|\mu(\operatorname{cl}(E) \backslash E)\|-\|\mu\|(F \backslash \operatorname{cl}(E)) \\
& \geqslant 2 \varepsilon-\varepsilon=\varepsilon .
\end{aligned}
$$

Suppose second that $\|\mu(\operatorname{cl}(E) \backslash E)\|<2 \varepsilon$. In this case we first specify a compact set $K \subset U \backslash \operatorname{cl}(E)$ such that $\|\mu(K)\| \geqslant 4 \varepsilon$, and we then obtain, as before, a set $F \in \mathscr{W}$ such that

$$
K \cup \operatorname{cl}(E) \subset \operatorname{int}(F) \subset \operatorname{cl}(F) \subset U,
$$

and such that

$$
\|\mu\|(F \backslash(K \cup \operatorname{cl}(E)))<\varepsilon .
$$

The obvious partitioning of $F \backslash E$ yields the inequalities

$$
\begin{aligned}
\|\mu(F \backslash E)\| & \geqslant\|\mu(K)\|-\|\mu(\operatorname{cl}(E) \backslash E)\|-\|\mu\|(F \backslash(K \cup \operatorname{cl}(E))) \\
& \geqslant 4 \varepsilon-2 \varepsilon-\varepsilon=\varepsilon .
\end{aligned}
$$

\section{Rosenthal's lemma revisited}

This section contains a variant of Rosenthal's lemma (Diestel and Uhl (1977), Lemma 1, p. 18) which applies directly to vector measures, but whose conclusions 
are slightly weaker than those of the original lemma. (This weakness will create only a slight inconvenience in the application of the lemma.) The interpolation property will come into play with a vengeance, and we isolate some technicalities into a preliminary lemma. This lemma clearly illustrates the role of the first four properties of a Wells class in making the interpolation property workable.

4.1. LEMMA. Let $\mathscr{W}$ be a Wells class, let $\left\{E_{n}\right\}$ be a topologically disjoint sequence in $\mathscr{W}$, let $\left\{D_{n}\right\}$ be a sequence of nonopen sets in $\mathscr{W}$ each of which is disjoint from $\bigcup_{n=1}^{\infty} E_{n}$, and let $\left\{K_{n}\right\}$ be a sequence of compact sets each of which is disjoint from $\operatorname{cl}\left(\bigcup_{n=1}^{\infty} E_{n}\right)$. Then there exists a set $E \in \mathscr{W}$ such that, for all $n$, we have $E_{n} \subset E \subset D_{n}^{c}$ and $\operatorname{cl}(E) \subset K_{n}^{c}$.

Proof. By property (1.1.3), we have $D_{n}^{c} \in \mathscr{W}$ for all $n$. By property (1.1.4) (via Lemma 1.3), there exists, for each $n$, a set $F_{n} \in \mathscr{W}$ such that

$$
\bigcup_{i=1}^{\infty} E_{i} \subset F_{n} \subset \operatorname{cl}\left(F_{n}\right) \subset K_{n}^{c} .
$$

Now property (1.1.1) can be used to convert the (countable) family of $D_{n}^{c}$ and $F_{n}$ into the decreasing sequence required for the interpolation property. The increasing sequence for the interpolation property will be

$$
\left\{E_{1}, E_{1} \cup E_{2}, E_{1} \cup E_{2} \cup E_{3}, \ldots\right\}
$$

and this is in $\mathscr{W}$ by property (1.1.2). The set $E \in \mathscr{W}$ which interpolates these sequences is clearly as desired. //

Here now is Rosenthal's lemma for regular Borel vector measures.

4.2. LEMma. Let $\mathscr{W}$ be a Wells class, let $\left\{E_{n}\right\}$ be a topologically disjoint sequence in $\mathscr{W}$, and let $\left\{\mu_{n}\right\}$ be a sequence of regular Borel vector measures. Then, for every number $\varepsilon>0$, there exists a subsequence $\left\{E_{n_{i}}\right\}$ and a set $E \in \mathscr{W}$ such that, for all $i$, we have:

(4.2.1) $E \supset E_{n_{i}}$; and

(4.2.2) $\left\|\mu_{n_{\ell}}\right\|\left(E \backslash \bigcup_{j=1}^{i} E_{n_{j}}\right)<\varepsilon$.

ProOF. Let $n_{1}=1$, and momentarily let $\mu=\mu_{n_{1}}$. Write $\bigcup_{n=2}^{\infty} E_{n}=\bigcup_{k=1}^{\infty} P_{k}$, where the sets $P_{k}$ are pairwise disjoint, and where each $P_{k}$ is the union of infinitely many of the $E_{n}$. Our immediate aim is to find, for each $k$, a set $G_{k} \in \mathscr{W}$ which will serve as an adequate substitute for $P_{k}$. To this end, we use the regularity of $\mu$ to obtain a sequence $\left\{K_{m}\right\}$ of compact sets such that each $K_{m}$ is disjoint from the set $K=\operatorname{cl}\left(\bigcup_{n=1}^{\infty} E_{n}\right)$, and such that the set $N=K^{c} \backslash \bigcup_{n=1}^{\infty} K_{n}$ is $\mu$-null. Also, using 
Zorn's lemma (if we are tired) or a standard exhaustion argument (if we are not tired), we obtain a (necessarily countable and possibly empty) maximal family $\left\{D_{m}\right\}$ of pairwise disjoint, non-open, nonnull sets in $\mathscr{W}$ such that each $D_{m}$ is disjoint from $\bigcup_{n=1}^{\infty} E_{n}$. In view of Lemma 4.1, we can now define $G_{k}$ to be any set in $\mathscr{W}$ such that $P_{k} \subset G_{k}$; such that $G_{k}$ is disjoint from each $D_{m}$; and such that $\operatorname{cl}\left(G_{k}\right)$ is disjoint from each $K_{m}$, and from each set of the form $\operatorname{cl}\left(E_{n}\right)$, where $E_{n} \cap P_{k}=\varnothing$.

We now argue that the sequence $\left\{G_{k}\right\}$ is essentially pairwise disjoint, and to this end we examine the set $G_{k} \cap G_{l}$ for distinct indices $k$ and $l$. This set, which lies in $\mathscr{W}$, is disjoint from each $E_{n}$ (since $P_{k} \cap P_{l}=\varnothing$ ). If it happens to be open, then it is also disjoint from $\operatorname{cl}\left(\bigcup_{n=1}^{\infty} E_{n}\right)$, and hence a subset of the null set $N$. If it is not open; then it must also be null, for otherwise the maximality of $\left\{D_{m}\right\}$ is violated. It follows that the standard disjointization procedure will not influence the $\mu$ measure of any $G_{k}$, so that, simply from the countable additivity of $\mu$, we may fix an index $k$ such that $\|\mu\|\left(G_{k}\right)<\varepsilon$.

Define $F_{1}=G_{k}$. Then we have:

(4.2.3) $\operatorname{cl}\left(F_{1}\right) \cap \mathrm{cl}\left(E_{n_{1}}\right)=\varnothing$;

(4.2.4) $\left\|\mu_{n_{1}}\right\|\left(F_{1}\right)<\varepsilon$; and

(4.2.5) $F_{1}$ contains infinitely many of the $E_{n}$.

Let $n_{2}$ be the smallest index such that $E_{n_{2}} \subset F_{1}$, and note that $n_{2}>n_{1}$. We now repeat the whole argument above with $n_{2}$ in place of $n_{1}$, and with the union of those $E_{n} \subset F_{1}$ such that $n \neq n_{2}$ in place of the set $\bigcup_{n=2}^{\infty} E_{n}$. The result will be a set $F_{2} \in \mathscr{W}$ which satisfies (4.2.3)-(4.2.5) in place of $F_{1}$ when $n_{1}$ is changed to $n_{2}$, and which, by (1.1.1), can be taken to be a subset of $F_{1}$.

As usual, we repeat the argument yet again, and we continue ad infinitum until sequences $\left\{E_{n_{i}}\right\}$ and $\left\{F_{i}\right\}$ in $\mathscr{W}$ have been produced such that, for each index $i$, we have:

(4.2.6) $\operatorname{cl}\left(F_{i}\right) \cap \operatorname{cl}\left(E_{n_{1}}\right)=\varnothing$;

(4.2.7) $\left\|\mu_{n_{i}}\right\|\left(F_{i}\right)<\varepsilon$; and

(4.2.8) $F_{i} \supset E_{n_{i+1}} \cup F_{i+1}$.

From here, one final direct application of the interpolation property will yield the desired set $E \in \mathscr{W}$ (compare with Wells (1969), p. 127). It is readily verified from properties (1.1.2), (4.2.6), and (4.2.8) that the sequences $\left\{\bigcup_{j=1}^{i} E_{n}\right\}_{i=1}^{\infty}$ and $\left\{F_{i} \cup\left(\bigcup_{j=1}^{i} E_{n_{j}}\right)\right\}_{i=1}^{\infty}$ satisfy the hypotheses of the interpolation property, and we choose $E \in \mathscr{W}$ to be any set which interpolates these sequences. That $E$ satisfies (4.2.1) is clear. That $E$ satisfies (4.2.2) is clear from (4.2.7) once we observe the containment $E \backslash \bigcup_{j=1}^{i} E_{n j} \subset F_{i}$. //

The above proof is more general than the lemma itself, and it can be adapted with considerable simplification to the more abstract setting. Here is the result. 
4.3. Lemma. Let $\mathscr{F}$ be a field (Boolean algebra) with the interpolation property, let $\left\{F_{n}\right\}$ be a pairwise disjoint sequence in $\mathscr{F}$, and let $\left\{\mu_{n}\right\}$ be a sequence of strongly (finitely) additive vector measures defined on $\mathscr{F}$ (Diestel and Uhl (1977), Definition 14 , p. 7). Then, for every number $\varepsilon>0$, there exists a subsequence $\left\{F_{n_{i}}\right\}$ and a set $F \in \mathscr{F}$ such that, for all $i$, we have:

(4.3.1) $F \supset F_{n i}$; and

(4.3.2) $\left\|\mu_{n_{i}}\right\|\left(F \backslash \mathbf{U}_{j=1}^{i} F_{n_{j}}\right)<\varepsilon$. //

Of course Lemma 4.3 can be used to obtain uniform boundedness principles in the abstract setting just as Lemma 4.2 will be used in the present setting. (And when $\mathscr{F}$ is a $\sigma$-algebra, the set $F$ is just $\bigcup_{i=1}^{\infty} F_{n_{i}}$ )

\section{The uniform boundedness principles}

We shall establish our two main results by direct sliding hump argumentsarguments which are similar in grand design but which are logically independent of one another. In this respect our approach differs from the standard approach in Diestel and Uhl (1977), wherein the Nikodym Boundedness Theorem (Theorem 1, p. 14) is used to prove the Nikodym Convergence Theorem (Theorem 8, p. 23). It also differs from the treatment of Wells (1969), who derives his boundedness theorem as a corollary (on p. 128) of his (unliberated) convergence theorem (our Corollary 5.3). With the aid of linear functionals, Wells' approach generalizes at once to this setting and, provided that one is prepared to employ a hefty dose of Banach space theory, it provides an attractive alternative to the present, more elementary approach.

Without further ado, here is our version of the Nikodym Boundedness Theorem.

5.1. TheOREM. Let $\mathscr{K}$ be a family of regular Borel vector measures on $\Omega$. Assume that there exists a Wells class $\mathscr{W}$ such that we have

$$
s_{E}=\sup \{\|\mu(E)\|: \mu \in \mathscr{K}\}<\infty
$$

for all $E \in \mathscr{W}$. Then $\mathscr{K}$ is uniformly bounded, that is

$$
\sup \{\|\mu\|(\Omega): \mu \in \mathscr{K}\}<\infty .
$$

Proof. Assume that the conclusion is false and (with no loss of generality) that $\mathscr{K}$ is countable. We shall obtain a topologically disjoint sequence $\left\{E_{n}\right\}$ in $\mathscr{W}$ and a sequence $\left\{\mu_{n}\right\}$ in $\mathscr{K}$ such that, for all $n \geqslant 2$, we have

$$
\left\|\mu_{n}\left(E_{n}\right)\right\| \geqslant n+\sum_{i=1}^{n-1}\left\|\mu_{n}\left(E_{i}\right)\right\| .
$$


An appeal to Lemma 4.2 (with $\varepsilon=1$, say) will produce a set $E \in \mathscr{W}$ on which the $\mu_{n}$ are unbounded. The arguments are standard (compare with Diestel and Uhl (1977), p. 16).

Here is the heart of our main construction: Let $K$ be a compact set with a 'do not enter' sign affixed, let $\rho>0$ be a preassigned constant, and assume that $\mathscr{K}$ is unbounded on a set $F \in \mathscr{W}$ whose closure is disjoint from $K$. Using the finiteness of $s_{F}$, we obtain, exactly as in Diestel and Uhl (1977), p. 15, a Borel set $B \subset F$ and a measure $\mu \in \mathscr{K}$ such that $\|\mu(B)\|>\rho$, and also such that $\|\mu(F \backslash B)\|>\rho$. Let $K_{1} \subset B$ and $K_{2} \subset F \backslash B$ be compact sets such that $\left\|\mu\left(K_{i}\right)\right\|>\rho$ for $i=1,2$, and then let $V_{1}$ and $V_{2}$ be disjoint open sets such that $K_{i} \subset V_{i}$ for $i=1,2$. We should like to get our hands on a compact set $H$ such that $\mathscr{K}$ is unbounded on $H$, and also such that $H$ is disjoint both from $K$ and also from $K_{i}$ for at least one value of $i$. To this end, we observe that $\mathscr{K}$ must be unbounded on at least one of the three sets $F \cap V_{1}, F \cap V_{2}$ and $F \cap\left(V_{1} \cup V_{2}\right)^{c}$, so that the desired set $H$ can be chosen to be of the form $\operatorname{cl}(F) \cap \operatorname{cl}\left(V_{i}\right)$, or of the form $\operatorname{cl}(F) \cap\left(V_{1} \cup V_{2}\right)^{c}$, as appropriate. Finally, we perform the normality-regularity stunt of Proposition 3.4 to specify an open set $U$ whose closure is disjoint from $K \cup H$, and to specify a set $E \in \mathscr{W}$ such that $K_{i} \subset E \subset \operatorname{cl}(E) \subset U$ (for the appropriate value of $i$ ), and such that $\|\mu(E)\| \geqslant \rho$.

We now weave this basic construction into a general induction procedure. Set $\mu_{n}=\mu$, set $E_{n}=E$, and set $U_{n}=U$. When $n=1$, let $K=\varnothing$, and otherwise let $K=\bigcup_{i=1}^{n-1} \operatorname{cl}\left(U_{i}\right)$ (where the previous sets $U_{i} \supset \operatorname{cl}\left(E_{i}\right)$ have been obtained by induction). The predetermined constant $\rho$ will be $=1$ when $n=1$, and it will be $=n+\sum_{i=1}^{n-1} s_{E_{i}}$ when $n>1$. Notice that the encasing of $\operatorname{cl}\left(E_{n}\right)$ in the open set $U_{n}$ (for all $n$ ) will guarantee the topological disjointness of the ultimate sequence $\left\{E_{n}\right\}$.

We are left with a new compact set $K^{*}=K \cup \operatorname{cl}\left(U_{n}\right)$ with its 'do not enter' sign affixed, and a compact set $H$, disjoint from $K^{*}$, on which $\mathscr{K}$ is unbounded. In order to continue the induction, and hence complete the proof, we must convert $H$ into an $F^{*} \in \mathscr{W}$ whose closure is disjoint from $K^{*}$, and on which $\mathscr{K}$ is unbounded. However, such a set $F^{*}$ is very simply obtained from the compactness of $H$ and from Lemma 1.3. //

Our version of the Nikodym Convergence Theorem follows immediately. Its proof appears easier only because some of the technicalities have been absorbed by Proposition 3.4.

5.2. THEOREM. Let $\left\{\mu_{n}\right\}$ be a sequence of regular Borel vector measures on $\Omega$. Assume that there exists a Wells class $\mathscr{W}$ such that the sequence $\left\{\mu_{n}(E)\right\}$ is convergent for each set $E \in \mathscr{W}$. Then

(5.2.1) the $\mu_{n}$ are uniformly countably additive; and 
(5.2.2) the limit $\mu(B)=\lim _{n \rightarrow \infty} \mu_{n}(B)$ exists for every set $B \in \mathscr{B}$; moreover, $\mu$ is a regular Borel measure.

Proof. We shall establish (5.2.1) under the assumption that $\lim _{n \rightarrow \infty} \mu_{n}(E)=0$ for all $E \in \mathscr{W}$. The general case follows exactly as in the proof of the standard theorem (Diestel and Uhl (1977), p. 24). By Proposition 3.4, it will suffice to verify criterion (3.4.1). If (3.4.1) fails, then, upon passing to a subsequence of $\left\{\mu_{n}\right\}$ if necessary, we may specify a number $\varepsilon>0$ and a topologically disjoint sequence $\left\{E_{n}\right\}$ in $\mathscr{W}$ such that $\left\|\mu_{n}\left(E_{n}\right)\right\| \geqslant 3 \varepsilon$ for all $n$. Since $\mu_{n}\left(E_{i}\right) \rightarrow 0$ for each fixed $i$, we may pass to a further subsequence, if necessary, in order to ensure that, for all $n \geqslant 2$, we have $\sum_{i=1}^{n-1}\left\|\mu_{n}\left(E_{i}\right)\right\|<\varepsilon$. By Lemma 4.2 , we may pass to a yet further subsequence, if necessary, in order to additionally ensure the existence of a set $E \in \mathscr{W}$ such that $E \supset \bigcup_{n=1}^{\infty} E_{n}$, and such that, for all $n$, we have

$$
\left\|\mu_{n}\right\|\left(E \backslash \bigcup_{i=1}^{n} E_{i}\right)<\varepsilon
$$

A contradiction now results from the inequalities

$$
\left\|\mu_{n}(E)\right\| \geqslant\left\|\mu_{n}\left(E_{n}\right)\right\|-\sum_{i=1}^{n-1}\left\|\mu_{n}\left(E_{i}\right)\right\|-\left\|\mu_{n}\right\|\left(E \backslash \bigcup_{i=1}^{n} E_{i}\right) \geqslant 3 \varepsilon-\varepsilon-\varepsilon=\varepsilon,
$$

which are valid for all $n \geqslant 2$.

The proof of (5.2.2) relies heavily upon the equivalence of uniform countable additivity and uniform regularity which was established in Proposition 3.4. Uniform regularity gives us two things: first, the regularity (and hence, by Proposition 3.1, the countable additivity) of the limit $\mu$, once it has been shown to exist; and second, via a standard $\varepsilon / 3$ argument, the fact that $\lim _{n \rightarrow \infty} \mu_{n}(K)$ exists for every compact set $K$. (Of course this $\varepsilon / 3$ argument will invoke the completeness of our nameless Banach space.) Via a similar $\varepsilon / 3$ argument, uniform countable additivity gives us the closure, under the formation of monotone limits, of the collection $\mathscr{L}$ of sets $B \in \mathscr{B}$ for which $\lim _{n \rightarrow \infty} \mu_{n}(B)$ exists. That $\mathscr{L}=\mathscr{B}$ now follows from the lemma on monotone classes (Halmos (1950), Theorem B, p. 27), provided that the way is cleared for the application of this lemma by the prior use of Exercises (2) and (3), pp. 25-26, in Halmos (1950). Alternatively, one may directly apply a less well-known variant of the lemma on monotone classes (Neveu (1965), Exercise I.4.4, p. 19). //

We shall not attempt to derive a maximal sequence of corollaries of these two theorems (but compare with Diestel and Uhl (1977), pp. 16ff, for an illustration of possible sorts of applications). Rather we simply make note of what are perhaps the most classical applications of this sort of result.

The first corollary generalizes (slightly) Wells' principal result (Wells (1969), 
Theorem 3, p. 125). For this corollary and the next, let $\mathscr{M}(\Omega)$ denote the Banach space of regular Borel scalar measures on $\Omega$, equipped with the total variation norm.

5.3. Corollary. A sequence $\left\{\mu_{n}\right\}$ in $\mathscr{M}(\Omega)$ converges weakly to an element of $\mathscr{M}(\Omega)$ if and only if there exists a Wells class $\mathscr{W}$ such that the sequence $\left\{\mu_{n}(E)\right\}$ is convergent for each set $E \in \mathscr{W}$.

Proof. Theorems 5.1 and 5.2 reduce this to the classical general result (Dunford and Schwartz (1958), Theorem 5, p. 308). //

The original version of the second corollary was due to Grothendieck (1953), Théorème 2, p. 146.

5.4. Corollary. A set $K \subset \mathscr{M}(\Omega)$ is relatively weakly (sequentially) compact if and only if there exists a Wells class $\mathscr{W}$ such that:

(5.4.1) $\sup \{\|\mu(E)\|: \mu \in \mathscr{K}\}<\infty$ for all $E \in \mathscr{W}$; and

(5.4.2) for every topologically disjoint sequence $\left\{E_{n}\right\}$ in $\mathscr{W}$, we have $\mu\left(E_{n}\right) \rightarrow 0$ uniformly for $\mu \in \mathscr{K}$.

Proof. Theorem 5.1 and criterion (3.4.1) reduce this to the classical general result (Dunford and Schwartz (1958), Theorem 1, p. 305). //

\section{References}

J. Diestel and J. J. Uhl, Jr. (1977), Vector measures (Mathematical Surveys 15, Amer. Math. Soc., Providence, R.I.).

N. Dinculeanu (1967), Vector measures (Internat. Series of Monographs in Pure and Appl. Math. 95, Pergamon Press, Oxford; VEB Deutscher Verlag der Wissenschaften, Berlin).

N. Dunford and J. T. Schwartz (1958), Linear operators: I. General theory (Pure and Appl. Math. 7, Interscience, New York).

A. Grothendieck (1953), 'Sur les applications linéaires faiblement compactes d'espaces du type $C(K)^{\prime}$, Canad.J. Math. 5, 129-173.

P. R. Halmos (1950), Measure theory (Van Nostrand, Princeton).

J. Neveu (1965), Mathematical foundations of the calculus of probability (Holden-Day, San Francisco).

G. L. Seever (1968), 'Measures on F-spaces', Trans. Amer. Math. Soc. 133, 267-280.

T. Traynor (1973), 'S-bounded additive set functions', in Vector and operator valued measures and applications, Proc. Sympos., Snowbird Resort, Alta, Utah (editors D. H. Tucker and H. B. Maynard), pp. 355-365 (Academic Press, New York).

B. B. Wells, Jr. (1969), 'Weak compactness of measures', Proc. Amer. Math. Soc. 20, 124-130.

Department of Mathematics

Monash University

Clayton, Victoria 3168

Australia 